\title{
The field brown dwarf LP 944-20 and the Castor moving group
}

\author{
I. Ribas ${ }^{\star}$ \\ Departament d'Astronomia i Meteorologia, Av. Diagonal, 647, 08028 Barcelona, Spain \\ Received 8 October 2002 / Accepted 23 December 2002

\begin{abstract}
A reliable age estimation for the field brown dwarf LP 944-20 is accomplished through the analysis of its kinematic properties. The space velocities of this star strongly suggest its membership in the so-called Castor moving group. LP 944-20 can be sensibly assumed to have the group's age, which is estimated to be $\sim 320 \mathrm{Myr}$, and metal content, which is found to be put to a test. Using the IR magnitudes and the lithium diagnostics, the models are able to provide a reasonable description of the brown dwarf's properties (to within a few sigma) but yield an age which is roughly $50 \%$ larger than our estimate. Possible reasons for this discrepancy are discussed.
\end{abstract} \\ roughly solar. With these new constrains and the available photometry and lithium abundance, current brown dwarf models are
}

Key words. stars: low-mass, brown dwarfs - stars: fundamental parameters - stars: kinematics - stars: individual: LP 944-20

\section{Introduction}

Brown dwarf (BD) research has made impressive progress after the first conclusive identification of a BD in 1995 (Nakajima et al. 1995). These objects, which populate the gap between low-mass stars and planets, are now observationally quite well characterized, especially with regard to their radiative and spectroscopic properties (see, e.g., Kirkpatrick et al. 1999; Basri 2000). Also, recent theoretical models, which most notably include dust formation and opacity, have evolved significantly and are thought to provide a realistic picture of BDs (Chabrier \& Baraffe 2000). However, some of the most important fundamental quantities, such as masses and ages, have not been directly determined for any of the bona-fide field BDs. Obviously, both mass and age are of crucial importance to assess the ability of theoretical models to make a trustworthy description of BD's properties. Only by limiting the number of free parameters in the comparison will we be able to critically analyze the performance of BD models. Unfortunately, masses can only be determined in a direct manner (i.e. dynamically) for BDs in binary systems, which are still very scarce. Age determination for BDs is even more challenging, and indirect indications from membership in open clusters or from stellar companions are the most sensible approaches.

Given the situation, one should take advantage of any opportunity to make reliable estimations of masses and ages of BDs. The field BD LP 944-20, as we show below, is one of the rare cases that allow for such estimation. The faint object LP 944-20, with spectral type M9 V, was first included in a high-proper motion star catalogue by Luyten \& Kowal (1975). It was not until much later that Tinney (1998; hereafter T98) first established its substellar nature through the so-called $\mathrm{Li}$

\footnotetext{
* e-mail: iribas@am.ub.es
}

test. During its short history as a confirmed BD, LP 944-20 has been observed at various wavelengths and holds the honour of being the first field substellar object to have been detected both in the radio and mid-IR domains (Berger et al. 2001; Apai et al. 2002). Also, LP 944-20 has been seen to experience X-ray flares but appears to have a low X-ray quiescent flux (Rutledge et al. 2000).

As a well-observed BD, LP 944-20 can serve as a valuable benchmark to evaluate the performance of theoretical models. To do so, one needs to constrain its physical properties as accurately as possible. Apparently LP 944-20, as an isolated object, would present a big challenge for the determination of its age. In this paper, we employ its kinematic properties to infer the object's age and metal content and use this information to carry out a test of current BD models.

\section{The kinematics of LP $944-20$ and the Castor moving group}

The space velocities $(U, V, W)$ of a star are readily computed from its position, proper motions, radial velocity and distance. In the case of LP 944-20, we have adopted the position, radial velocity and distance from Tinney (1996) and T98. In addition, values for the proper motions have been provided by Luyten (1979) and Tinney (1996). Surprisingly, the difference between these two values is very large, amounting to over $10 \sigma$. Since the effect on the computed space velocities is fairly small, we will not discuss possible reasons for the discrepancy, but the large parallax ( 200 mas) of LP 944-20 or a typographical error might be responsible. Instead of favoring one of the values, we have listed the space velocities resulting from the two different values of the proper motion in Table 1 (Case I: Tinney 1996; Case II: Luyten 1979). Note that we have followed the 
Table 1. Astrometric and kinematic data for LP 944-20. Space velocities in Case I have been computed using the proper motion in Tinney (1996), while Case II makes use of the proper motion in the NLTT Catalogue of Luyten (1979).

\begin{tabular}{lcc}
\hline \hline \multicolumn{1}{c}{ Parameter } & Case I & Case II \\
\hline$\mu_{\alpha} \cos \delta\left(\mathrm{mas} \mathrm{yr}^{-1}\right)$ & 324.0 & 210.2 \\
$\mu_{\delta}\left(\mathrm{mas} \mathrm{yr}^{-1}\right)$ & 295.9 & 259.6 \\
$d(\mathrm{pc})$ & $5.04 \pm 0.11$ & $5.04 \pm 0.11$ \\
$v_{\mathrm{r}}\left(\mathrm{km} \mathrm{s}^{-1}\right)$ & $10.0 \pm 2.0$ & $10.0 \pm 2.0$ \\
$U\left(\mathrm{~km} \mathrm{~s}^{-1}\right)$ & $-12.6 \pm 0.8$ & $-10.7 \pm 0.8$ \\
$V\left(\mathrm{~km} \mathrm{~s}^{-1}\right)$ & $-6.1 \pm 1.4$ & $-4.8 \pm 1.4$ \\
$W\left(\mathrm{~km} \mathrm{~s}^{-1}\right)$ & $-3.5 \pm 1.9$ & $-5.1 \pm 1.9$ \\
\hline
\end{tabular}

convention where positive values of $U, V$, and $W$ indicate velocities towards the galactic center, galactic rotation and north galactic pole, respectively.

The existence of a group of stars kinematically linked to the sextuple system Castor was first proposed by Anosova \& Orlov (1991). Some 18 stars with spectral types between A1 V and M6 Ve were suggested to be members of the moving group. Barrado y Navascués (1998) later revisited the situation and provided a new list of Castor moving group candidates based upon more restrictive criteria such as kinematics, position in H-R diagram and lithium abundance. Barrado y Navascués lists a total of 17 possible members of the moving group. More recently, Montes et al. (2001) proposed the membership of several additional late type stars in the moving group using purely kinematic criteria. With these lists as starting point, we have performed a search in the literature and used our own observations to compile VRI photometry. The measurements are presented in Table 2. We have further completed the list by adding the components of the quadruple system GJ 2069, which also share the space velocities of the group members. The implications of the GJ 2069 membership in the moving group are discussed in Ribas (2003).

A color magnitude plot of all stars in Table 2 is shown in Fig. 1. Also plotted are two isochrones for the estimated age of the moving group (see next section) computed using the models by Siess et al. (2000) and Baraffe et al. (1998). Using this diagram we have further refined the membership list by isolating and eventually excluding three stars that appear to deviate significantly above the isochrone (empty circles in Fig. 1). Note that this criterion can only be applied to stars that are massive enough (i.e. early spectral types) to experience evolutionary changes in timescales of a few 100 Myr. All the excluded stars have luminosity class IV, which agrees with our claim that these are older and more evolved than the Castor moving group members. The agreement of the theoretical isochrones and the sequence defined by the stars hotter than spectral class $M$ is excellent. One of the deviating objects with the earliest spectral type is the double-lined eclipsing binary YY Gem (G1 $278 \mathrm{C}$ ), which is composed of a pair of M1 V stars, and was analyzed in detail by Torres \& Ribas (2002). The authors found that current stellar models fail to reproduce the observed physical properties of the stars by up to $20 \%$. This same situation is also likely
Table 2. Photometry for the Castor moving group members in Barrado y Navascués (1998) and Montes et al. (2001) with additional members suggested in this work.

\begin{tabular}{|c|c|c|c|c|c|c|}
\hline Name & SP & $V$ & $I_{\mathrm{C}}$ & $(R-I)_{\mathrm{C}}$ & $d(\mathrm{pc})$ & $M_{\mathrm{I}}$ \\
\hline G1 20 & A7 V & 3.933 & 3.734 & 0.096 & 23.5 & 1.877 \\
\hline Gl 217.1 & A2 Vann & 3.542 & 3.436 & 0.049 & 21.5 & 1.772 \\
\hline G1 226.2 & K8 V & 9.75 & 8.26 & 0.66 & 24.9 & 6.276 \\
\hline Gl 255 A & F8 IV-V & 6.795 & 6.28 & 0.250 & 43.2 & 3.10 \\
\hline G1 255 B & - & 7.209 & 6.67 & 0.265 & 43.2 & 3.49 \\
\hline Gl $278 \mathrm{~A}$ & $\mathrm{~A} 1 \mathrm{~V}$ & 1.93 & 1.915 & 0.008 & 14.9 & 1.042 \\
\hline Gl 278 B & $\mathrm{A} 5 \mathrm{Vm}$ & 2.93 & 2.775 & 0.084 & 14.9 & 1.902 \\
\hline Gl $278 \mathrm{C}$ & M1 V & 9.074 & 7.155 & 0.993 & 14.9 & $7.035^{1}$ \\
\hline Gl $351 \mathrm{~A}^{2}$ & F2 IV & 3.887 & 3.454 & 0.214 & 18.6 & 2.111 \\
\hline GJ $521.2 \mathrm{~A}$ & F7.7 V & 6.34 & 5.86: & $0.22:$ & 25.2 & 3.85: \\
\hline Gl $564.1^{2}$ & A3 IV & 2.747 & 2.591 & 0.078 & 23.7 & 0.720 \\
\hline G1 721 & $\mathrm{~A} 0 \mathrm{~V}$ & 0.034 & 0.039 & 0.004 & 7.8 & 0.590 \\
\hline Gl 755 & G5 V & 6.477 & 5.792 & 0.335 & 20.9 & 4.195 \\
\hline Gl $826^{2}$ & A7 IV-V & 2.459 & 2.195 & 0.120 & 15.0 & 1.320 \\
\hline GJ 842.2 & M0.5 V & 10.57 & 8.69 & 0.97 & 20.9 & 7.09 \\
\hline G1 879 & K4 V & 6.485 & 5.283 & 0.540 & 7.6 & 5.868 \\
\hline Gl 881 & A3 V & 1.155 & 1.077 & 0.025 & 7.7 & 1.647 \\
\hline Gl 896 A & M3.5 V & 10.26 & 7.635 & 1.516 & 6.2 & $8.8^{1}$ \\
\hline G1 896 B & $\mathrm{M} 4.5 \mathrm{~V}$ & 12.47 & 9.296 & 1.873 & 6.21 & $10.45^{1}$ \\
\hline GJ 1111 & M6.5 V & 14.79 & 10.53 & 2.26 & 3.61 & 12.73 \\
\hline GJ $2069 \mathrm{Aa}$ & M3.5 V & 12.49 & 9.71 & 1.59 & 12.8 & 9.17 \\
\hline GJ $2069 \mathrm{Ab}$ & M3.5 V & 12.82 & 9.99 & 1.62 & 12.8 & 9.45 \\
\hline GJ $2069 \mathrm{Ba}$ & $\mathrm{M} 4.5 \mathrm{~V}$ & 13.60 & 10.68 & 1.66 & 12.81 & 10.14 \\
\hline GJ $2069 \mathrm{Bb}$ & M4.5 V & 14.94 & 11.63 & 1.93 & 12.81 & 11.09 \\
\hline GJ 3633 & K0 V & 7.34 & - & - & 17.5 & - \\
\hline HD 77825 & $\mathrm{~K} 2 \mathrm{~V}$ & 8.78 & 7.82 & 0.48 & 28.1 & 5.58 \\
\hline LP 944-20 & M9 V & 19.2: & 14.16 & 2.94 & $5.0 \quad 1$ & 15.65 \\
\hline
\end{tabular}

${ }^{1}$ Corrected for duplicity.

${ }^{2}$ Possibly not a member. See text.

to occur for later spectral types. Thus, the systematic deviation that arises for spectral types $\mathrm{M}$ is not surprising.

With the updated list of members and the individual velocities in Barrado y Navascués (1998) and Montes et al. (2001) we have computed mean velocities for the moving group, which are $\langle U\rangle=-10.6 \pm 3.7 \mathrm{~km} \mathrm{~s}^{-1},\langle V\rangle=-6.8 \pm 2.3 \mathrm{~km} \mathrm{~s}^{-1}$, and $\langle W\rangle=-9.4 \pm 2.1 \mathrm{~km} \mathrm{~s}^{-1}$, i.e. $(-10.6,-6.8,-9.4) \mathrm{km} \mathrm{s}^{-1}$. The velocities that we find for LP $944-20$ (see Table 1) are in good agreement with the mean values for the Castor moving group. The most discrepant velocity component is $W$, with a difference of 4-6 $\mathrm{km} \mathrm{s}^{-1}$ but still marginally compatible within the error bars. Both $U$ and $V$ velocities are well within the uncertainties of the mean values found for the moving group. Also, we report that LP 944-20 fulfills the two moving group membership criteria described in Montes et al. (2001). The total space velocity difference between LP 944-20 and the Castor moving group average is $4.7-6.3 \mathrm{~km} \mathrm{~s}^{-1}$ (depending on the velocities adopted for LP 944-20; see Table 1). This difference is consistent with the membership of LP 944-20 in the Castor moving group given its relatively old age (see Sect. 3) and the expected peculiar velocities of the members due to disk heating. A work with similar goals to ours was carried out by Ruiz et al. (1991), who studied the membership of the BD candidate 


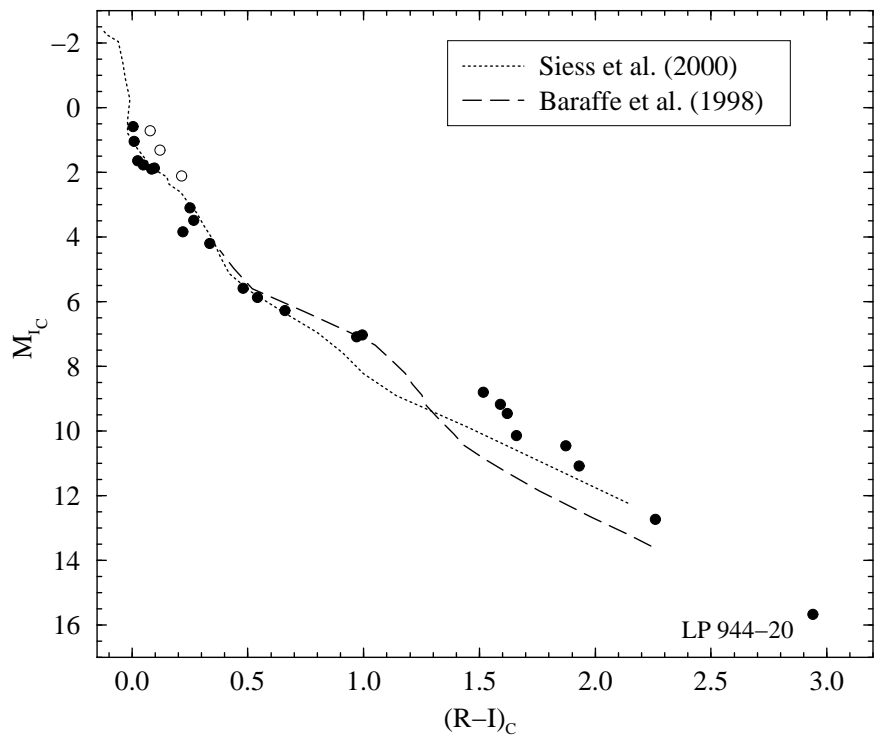

Fig. 1. Color-magnitude diagram for the Castor moving group members with $R$ and $I$ photometry. Also plotted are two isochrones with an age of 370 Myr computed using the models of Baraffe et al. (1998) and Siess et al. (2000). Three early-type stars that deviate significantly from the isochrone are represented by empty circles and possibly do not belong in the moving group (see Table 2 for identification). The systematic deviations for $(R-I)$ colors greater than 1 (M stars) are likely to arise from shortcomings of current atmosphere models (see text for further information).

ESO 207-61 in the Hyades moving group. The difference of space velocities between the BD candidate and the mean velocity of the moving group was found to be over $9 \mathrm{~km} \mathrm{~s}^{-1}$, yet these authors concluded that ESO 207-61 was a likely member of the Hyades moving group. The case for LP 944-20 is even stronger.

Several studies have been published in recent years (Sabas 1997; Dehnen 1998; Asiain et al. 1999) dealing with the kinematic analysis of large stellar samples, which have led to the detection of numerous moving groups. Interestingly, all these studies report stellar kinematic groups with velocities very similar to those we find for the Castor moving group: Group "D" found by Sabas (1997) has velocity components $(-12.5,-6.2,-8.3) \mathrm{km} \mathrm{s}^{-1}$, Dehnen (1998) gives $(-10,-5,-8) \mathrm{km} \mathrm{s}^{-1}$ for his Group 4, and Asiain et al. (1999) obtain $(-10.5,-5.0,-7.5) \mathrm{km} \mathrm{s}^{-1}$ for their group "C1". All these, often referred to as the Coma Berenices group, can possibly be identified as the Castor moving group with the small velocity differences arising from the various stellar samples considered and analysis methods used. Interestingly, the Castor moving group has quite different velocities to those of all the other young (age < $600 \mathrm{Myr}$ ) kinematic groups reported by these publications (see also Montes et al. 2001). Thus, the similarity (within $1 \sigma$ ) between the velocities of LP 944-20 and those listed above reduces the possibility of misclassification strengthens our case for the membership of LP 944-20 in the Castor moving group.

\section{The age and metallicity of LP $944-20$}

LP 944-20 became one of the first bona-fide BDs when T98 reported the detection of lithium in the spectrum, thus confirming its substellar nature. T98 compared the equivalent width of the $\mathrm{Li}$ I $\lambda 6708$ line with theoretical models and estimated an age between 475 and $650 \mathrm{Myr}$, and a mass of $0.056-0.064 M_{\odot}$. Other independent tests seemed to roughly confirm an intermediate age for this object.

The membership of LP 944-20 in the Castor moving group discussed in the previous section opens a new perspective. Indeed, it is sensible to assume that LP 944-20 has the same age and chemical composition as the rest of the group members - similarly to what is routinely done in stellar cluster studies. Barrado y Navascués (1998) used criteria based on kinematics, isochrone fitting, and lithium abundances to estimate the age of the moving group to be $200 \pm 100$ Myr. Asiain et al. (1999) estimated the age of their "C1" group (which we identify as the Castor moving group) from isochronal fits and obtained $400 \pm 200$ Myr. Using a very similar procedure, Sabas (1997) derived an age of $320 \pm 150$ Myr for her group "D" (also identified as the Castor moving group). The study by Torres \& Ribas (2002) analyzed in detail the Castor sextuple system and obtained an age of $370 \pm 50 \mathrm{Myr}$ and a nearly solar value for the metallicity. A further independent estimate is possible through the study of the activity level of the solar analog G1 755 (spectral type G5 V). Stars narrowly confined in spectral type between G0 V and G5 V have well studied correlations between their X-ray luminosities and ages (see Dorren et al. 1995). Using the X-ray luminosity for Gl 755 in Hünsch et al. (1999) and the relationship of Güdel et al. (1997) we derive a lower limit for its age of $200 \mathrm{Myr}$. Note that only a lower limit can be obtained because Gl 755 is a poorly-understood close binary and a fraction of the X-ray emission could be coming from an unseen companion (Gaidos 1998).

With these considerations in mind, we have adopted an age of $320 \pm 80 \mathrm{Myr}$ and a metal content of $Z=Z_{\odot}$ for the moving group members. Our value for the age is therefore lower than the model-dependent estimate by T98. Even though sometimes overlooked, chemical composition is also an important parameter that has a critical influence on the evolution of stars and BDs. Not only we have just obtained an independent estimate of the age of LP 944-20 but also we have information on its metallicity. Under these circumstances a test of current BD models is possible. Also, the estimated age of LP 944-20 is nicely placed between the Pleiades and Hyades and allows for an analysis of models in an age domain so far unexplored.

\section{Comparison with brown dwarf models}

Several theoretical models for substellar masses are currently available in the literature. These include the models of D'Antona \& Mazzitelli (1994), Burrows et al. (1997), Baraffe et al. (1998), and Chabrier et al. (2000). With both the age and the metal content known, the mass and temperature of LP 944-20 can be readily estimated by interpolating these models at, for instance, the observed bolometric luminosity (to avoid employing uncertain color transformations for the time 
Table 3. Red and IR photometry for LP 944-20. The transformation from the 2MASS to the CIT systems has been carried out by using expressions in Carpenter (2001).

\begin{tabular}{lrlr}
\hline \hline \multicolumn{4}{c}{ Red and IR photometry $(\mathrm{mag})$} \\
\hline$J_{\text {2MASS }}=$ & $10.75 \pm 0.03$ & $I_{\mathrm{C}}=$ & $14.16 \pm 0.04$ \\
$H_{2 \text { MASS }}=$ & $10.02 \pm 0.03$ & $(m-M)=$ & $-1.49 \pm 0.05$ \\
$K_{\text {S2MASS }}=$ & $9.52 \pm 0.03$ & $M_{\text {IC }}=$ & $15.65 \pm 0.04$ \\
$J_{\text {CIT }}=$ & $10.72 \pm 0.03$ & $M_{\text {KCIT }}=$ & $11.04 \pm 0.06$ \\
$H_{\text {CIT }}=$ & $10.00 \pm 0.03$ & $\left(I_{\mathrm{C}}-K_{\mathrm{CIT}}\right)=$ & $4.61 \pm 0.05$ \\
$K_{\mathrm{CIT}}=$ & $9.55 \pm 0.03$ & & \\
\hline
\end{tabular}

Table 4. Mass and effective temperature derived from the observed age and luminosity of LP 944-20 for several BD models. The interpolation parameter was the bolometric luminosity.

\begin{tabular}{lll}
\hline \hline \multicolumn{1}{c}{ Models } & \multicolumn{1}{c}{ Mass $\left(M_{\odot}\right)$} & \multicolumn{1}{c}{$T_{\text {eff }}(\mathrm{K})$} \\
\hline D’Antona \& Mazzitelli (1994) & 0.055 & 2060 \\
Burrows et al. (1997) & $0.050-0.055$ & $2000-2100$ \\
Baraffe et al. (1998) & 0.049 & 2000 \\
Chabrier et al. (2000) & 0.049 & 1970 \\
\hline
\end{tabular}

being). The luminosity of LP 944-20 follows from its absolute $K$ magnitude (in Table 3 ) and a bolometric correction $\left(B C_{K 2 \mathrm{MASS}}=+3.21 \pm 0.07 \mathrm{mag}\right)$ computed using the atmosphere models by Allard et al. (2001). We obtained a value of $\log \left(L / L_{\odot}\right)=-3.79 \pm 0.04$. Interpolation in the model grids yielded values for the mass and effective temperature presented in Table 4 . Mass estimates cluster around $0.05 M_{\odot}$ with a scatter of about $6 \%$ and the effective temperature comes out to be nearly $2000 \mathrm{~K}$ with a scatter of $2.5 \%$. Note that this value is significantly smaller than the effective temperature estimated by Basri et al. (2000) for LP 944-20, which was found to be $T_{\text {eff }} \approx 2400 \mathrm{~K}$ from spectral line fits. Also, Luhman (1999) assigns an effective temperature of $2400 \mathrm{~K}$ to dwarf M 9 objects by extrapolating the scale of Leggett et al. (1996). These results suggest the existence of significant disagreements between the effective temperatures predicted by the models and the empirical scales. Our value for the mass is somewhat lower than the estimate by T98 who obtained $0.056-0.064 M_{\odot}$ using similar BD models. The reason for this higher mass can be found in the larger value for the age adopted by T98.

After this rough comparison with model data, we proceeded one step further and performed a more critical test of models using the available photometric information. The recent BD models of Chabrier et al. (2000) were employed for a detailed comparison because these provide a complete set of optical/IR magnitudes, lithium abundances and bolometric luminosities for a range of masses and ages. Also these models incorporate modern input physics and atmospheres that include dust formation.

The available data for LP 944-20 to perform a comparison with models are accurate IR magnitudes and colors, and a measurement of the Li I $\lambda 6708$ equivalent width. IR photometry of LP 944-20 is available from the 2MASS Point Source Catalogue Second Incremental Release (Skrutskie et al. 1997)

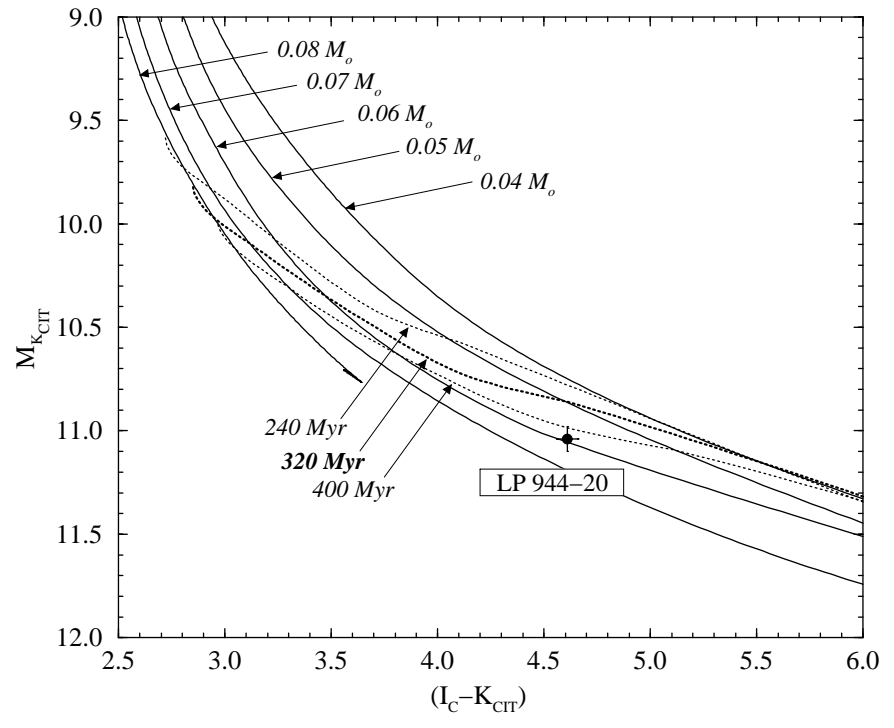

Fig. 2. Color-magnitude diagram at the BD regime. The observational data point for LP 944-20 is shown. Evolution tracks (solid lines) and isochrones (dotted lines) were interpolated from the model grid of Chabrier et al. (2000).

and is presented in Table 3. Also in the table are the IR magnitudes transformed to the CIT system using expressions in Carpenter (2001). This was done because photometry of BD models in Chabrier et al. (2000) is given in the CIT system.

Figure 2 presents a color magnitude diagram at the bottom of the main sequence. The data point with error bars corresponds to the photometry of LP 944-20 with its uncertainty. Evolution tracks for several masses computed by interpolating in the model grids of Chabrier et al. (2000) are represented with solid lines. Isochrones for the estimated age of LP 944-20 and $\pm 1 \sigma$ were also interpolated from the models and are shown on the plot as dotted lines. As can be seen, the observed photometry for LP 944-20 is just marginally compatible with the model predictions at the upper limit for the age (400 Myr) and some $2.5 \sigma$ below the predicted $K$ magnitude at the adopted age (320 Myr). Using the photometry alone, BD models would overestimate the age by predicting a value of about $500 \mathrm{Myr}$ (and a mass of $0.06 M_{\odot}$ ). This result indicates that, while models provide a reasonable general description of the BD's radiative properties, a closer look indicates that a photometry-based determination of the age for LP 944-20 may be overestimated by as much as $50 \%$.

A complementary test of models follows from the comparison of the predicted and observed lithium abundances. As it is well known, the lithium test has become one of the primary diagnostics to identify an object as a BD (see Basri 2000 for a review). The test is especially useful for candidates of spectral type later than M 7 because lithium would be completely depleted should they not be BDs. This is the case of LP 944-20, with a spectral type of M 9, where the detection of the $\mathrm{Li}$ I $\lambda 6708$ line was reported by T98. However, lithium is also eventually destroyed in the more massive BDs when their central temperatures become sufficiently high. The sole detection of lithium in LP 944-20 (and with its known luminosity) proves that its mass is substellar and provides 
(model-dependent) upper limits to both the age ( $\leq 650 \mathrm{Myr}$ ) and the mass $\left(\leq 0.065 M_{\odot}\right)$ of the object.

More quantitatively, the $\mathrm{Li}$ I $\lambda 6708$ equivalent width measured by $\mathrm{T} 98$ seems to indicate that lithium has already been partially depleted in LP 944-20, with a current abundance ratio of $\log \left[n(\mathrm{Li}) / n_{\circ}(\mathrm{Li})\right]=-2.5 \pm 1.0$. Using our estimate for the age $(320 \pm 80 \mathrm{Myr})$ and interpolating in the model grids of Chabrier et al. (2000) leads to a mass of $0.063 \pm 0.001 M_{\odot}$ and a bolometric luminosity of $\log \left(L / L_{\odot}\right)=-3.50 \pm 0.14$. Note that this latter value is inconsistent with the observed luminosity of $\log \left(L / L_{\odot}\right)=-3.79 \pm 0.04$. Turning the argument around, when both the observed lithium abundance and luminosity are used to compare with model predictions one obtains an age of $490 \pm 100 \mathrm{Myr}$ and a mass of $0.061 \pm 0.005 M_{\odot}$. This was the exact same procedure used by T98, as illustrated in his Fig. 2. Again, the results obtained from the lithium abundance seem to overestimate the age of LP $944-20$ by about $50 \%$.

The apparent inconsistency found when comparing the known age of LP 944-20 with estimates from photometry is not surprising. While atmospheres of very low mass objects have come a long way, there is still room for improvement and it is likely that broad band magnitudes and colors can be refined. In this sense, our result for LP 944-20 may prove useful as it can define an anchor point for intermediate age BDs The differences detected when using the observed lithium abundances are somewhat more disturbing. The lithium depletion mechanism is simple and apparently well understood. At least four scenarios can be put forward to explain the observed discrepancy: 1) An underestimation of the measured lithium abundances. LP 944-20 is a relatively fast rotator $\left(v \sin i=30 \pm 2.5 \mathrm{~km} \mathrm{~s}^{-1}\right.$; Basri et al. 2000) and the spectrum acquired by T98 has fairly low $S / N$, which could have resulted in an underestimated equivalent width. 2) Current BD models underestimate the lithium destruction rate. Rotation, which is not currently accounted for by evolution models, could play an important role (Martín \& Claret 1996). A tantalizing hint that favors this scenario is the nearly constant $50 \%$ age differential found between the "classical" ages of young clusters and the lithium-based determinations (see Basri 2000 for a summary). This is the exact same differential we find for LP 944-20. 3) Dobbie et al. (2002) have recently suggested that theoretical models might systematically underestimate $\mathrm{BD}$ masses. Both the temperature and $\mathrm{Li}$ abundance discrepancies described above could be diminished or resolved if BD masses predicted by models were larger. 4) Obviously, a possibility still remains that LP 944-20 is not a member of the Castor moving group and that its age and mass are indeed around $500 \mathrm{Myr}$ and $0.06 M_{\odot}$, respectively. This cannot be ruled out completely because the velocities of the Castor moving group are quite similar to those of the Local Standard of Rest (Dehnen \& Binney 1998).

\section{Conclusions}

In this paper we exploit the probable membership of the lithium BD LP 944-20 in the Castor moving group. Two otherwise unknown parameters, the age and metal content, can be obtained for the moving group from a variety of methods (isochrones, activity-age relationship, lithium abundance measurements, etc) and then adopted for LP 944-20. Very importantly, the knowledge of the age and metallicity reduces the number of free parameters and a critical test of BD models becomes possible. Our results show that models are able to reproduce the observed properties of LP 944-20 to within a few sigma, but some discrepancies were detected. In particular, the analysis of the IR color-magnitude diagram revealed that, with current atmosphere models, the age of LP 944-20 obtained from this diagnostic would be overestimated by as much as 50\%. A similar result was found when considering the age predicted by the models when using the measured lithium abundance.

Our age estimate for LP 944-20 not only serves as a constraint to evaluate theoretical models but it also bears on the question of the formation mechanism of BDs. Apai et al. (2002) recently reported the non detection of mid-IR excess in LP 944-20 thus indicating the lack of warm dust around it. The new age we have determined sets a tight constraint on the disk dissipation time that $\mathrm{BD}$ formation models will have to address.

The Castor moving group could be very important because most of its members lie within 5-20 pc of the Sun. As a result, the main sequence of the group can be relatively easily followed down to very low masses. As an intermediate-age association (320 Myr), the Castor moving group can prove very useful to study BDs in an evolutionary stage beyond that of the Pleiades objects. With this goal in mind, we are currently investigating the possible membership of other nearby BD candidates in this moving group. A very promising object is the M8.5 dwarf 2MASSI J0335020+234235 for which Reid et al. (2002) determined space velocities of $U=-13.0 \pm 1.3 \mathrm{~km} \mathrm{~s}^{-1}$, $V=-4.8 \pm 0.4 \mathrm{~km} \mathrm{~s}^{-1}$, and $W=-4.7 \pm 0.1 \mathrm{~km} \mathrm{~s}^{-1}$. These velocities are very similar to those of LP 944-20 and the Castor moving group average (see Table 1 and Sect. 2). Interestingly, Reid et al. reported the detection of lithium in 2MASSI J0335020+234235 thus proving its BD status. According to our results, this BD could also have an age of about 320 Myr. The analysis of this and further Castor moving group BDs should be able to shed some light on the age discrepancy found for LP 944-20. Furthermore, we are also extending the analysis to other young, nearby moving groups that will permit the generalization of the study to BDs with a variety of ages and chemical compositions.

Acknowledgements. I am indebted to R. Rebolo, who suggested the idea of attempting a kinematic age determination for LP 944-20. I gratefully acknowledge F. Figueras, D. Fernández and C. Jordi for fruitful discussions, and the referee, R. F. Jameson, for a number of constructive and useful comments. This publication makes use of data products from the Two Micron All Sky Survey, which is a joint project of the University of Massachusetts and the Infrared Processing and Analysis Center/California Institute of Technology, funded by the National Aeronautics and Space Administration and the National Science Foundation. This research has made use of the SIMBAD database, operated at CDS, Strasbourg, France. This research has made use of NASA's Astrophysics Data System. 


\section{References}

Allard, F., Hauschildt, P. H., Alexander, D. R., Tamanai, A., \& Schweitzer, A. 2001, ApJ, 556, 357

Anosova, J. P., \& Orlov, V. V. 1991, A\&A, 252, 123

Asiain, R., Figueras, F., Torra, J., \& Chen, B. 1999, A\&A, 341, 427

Apai, D., Pascucci, I., Henning, Th., et al. 2002, ApJ, 573, L115

Baraffe, I., Chabrier, G., Allard, F., \& Hauschildt, P. H. 1998, A\&A, 337,403

Barrado y Navascués, D. 1998, A\&A, 339, 831

Basri, G. 2000, ARA\&A, 38, 485

Basri, G., Mohanty, S., Allard, F., et al. 2000, ApJ, 538, 363

Berger, E., Ball, S., Becker, K. M., et al. 2001, Nature, 410, 338

Burrows, A., Marley, M., Hubbard, W. B., et al. 1997, ApJ, 491, 856

Carpenter, J. 2001, AJ, 121, 2851

Chabrier, G., \& Baraffe, I. 2000, ARA\&A, 38, 337

Chabrier, G., Baraffe, I., Allard, F., \& Hauschildt, P. H. 2000, ApJ, 542,464

D’Antona, F., \& Mazzitelli, I. 1994, ApJS, 90, 467

Dehnen, W. 1998, AJ, 115, 2384

Dehnen, W., \& Binney, J. J. 1998, MNRAS, 298, 387

Dobbie, P. D., Pinfield, D. J., Jameson, R. F., \& Hodgkin, S. T. 2002, MNRAS, 335, L79

Dorren, J. D., Güdel, M., \& Guinan, E. F. 1995, ApJ, 448, 431

Gaidos, E. J. 1998, PASP, 110, 1259

Güdel, M., Guinan, E. F., \& Skinner, S. L. 1997, ApJ, 483, 947

Hünsch, M., Schmitt, J. H. M. M., Sterzik, M. F., \& Voges, W. 1999, A\&AS, 135, 319

Kirkpatrick, J. D., Reid, I. N., Liebert, J., et al. 1999, ApJ, 519, 802
Leggett, S. K., Allard, F., Berriman, G., Dahn, C. C., \& Hauschildt, P. H. 1996, ApJS, 104, 117

Luhman, K. L. 1999, ApJ, 525, 466

Luyten, W. J. 1979, LHS catalogue. A catalogue of stars with proper motions exceeding 0'.5 annually (University of Minnesota, Minneapolis)

Luyten, W. J., \& Kowal, C. T. 1975, Proper Motion Survey with the 48 inch Schmidt Telescope. XLIII. One Hundred and Six Faint Stars with Large Proper Motion (University of Minnesota, Minneapolis)

Martín, E., \& Claret, A. 1996, A\&A, 306, 408

Montes, D., López-Santiago, J., Gálvez, M. C., et al. 2001, MNRAS, 328,45

Nakajima, T., Oppenheimer, B. R., Kulkarni, S. R., et al. 1995, Nature, 378, 463

Reid, I. N., Kirkpatrick, J. D., Liebert, J., et al. 2002, AJ, 124, 519

Ribas, I. 2003, A\&A, 398, 239

Ruiz, M. T., Takamiya, M. Y., \& Roth, M. 1991, ApJ, 367, L59

Rutledge, R. E., Basri, G., Martín, E. L., \& Bildsten, L. 2000, ApJ, 538, L141

Sabas, V. 1997, Ph.D. Thesis, Obs. de Paris

Siess, L., Dufour, E., \& Forestini, M. 2000, A\&A, 358, 593

Skrutskie, M. F., Schneider, S. E., Stiening, R., et al. 1997, in The Impact of Large Scale Near-IR Sky Surveys, ed. F. Garzón et al. (Dordrecht: Kluwer), 25

Tinney, C. G. 1996, MNRAS, 281, 644

Tinney, C. G. 1998, MNRAS, 296, L42 (T98)

Torres, G., \& Ribas, I. 2002, ApJ, 567, 1140 